\title{
Energiestoffwechsel und Serumenzyme
}

\author{
Von H. KRÖNER und W. STATB \\ Aus dem Physiologisch-chemischen Institut der Universität Dïsseldorf (Direktor: Prof. Dr. S. Hollmann)
}

(Eingegangen am 2. August 1966)

In verschiedenen zeitlichen Abständen nach intraperitonealer Injektion von Monojodacetat und 2,4-Dinitrophenol wurden die Adeninnucleotide sowie Lactat und Pyruvat in der Rattenleber bestimmt. Das Verhältnis der Adeninnucleotide als Maß für die freie Energie der Zelle wird dabei sehr unterschiedlich verschoben. Unter den gleichen Versuchsbedingungen wurden die Aktivitäten verschiedener Serumenzyme gemessen. In vielen Fällen sind diese zwar crhöht, jedoch ist die Zunahme nur relativ gering. Ein möglicher kausaler Zusammenhang wird diskutiert.

Adenine nucleotides, lactate and pyruvate were determined in rat liver at different time intervals after the intraperitoneal injection of monoiodoacetate and 2.4-dinitrophenol. The relative levels of the adenine nucleotides as a measure of the free energy of the cell were considerably changed. The activities of various enzymes were measured under the same experimental conditions. These were increased in many cases, but the increase was relatively small. A possible causal relationship is discussed.

Sowohl aufgrund klinischer Beobachtungen als auch aufgrund zahlreicher experimenteller Befunde $(1-4)$ ist bekannt, da $\beta$ mangelnde Sauerstoffversorgung von $\mathrm{Ge}$ weben zum Ausstrom von Enzymen aus der Zelle in die Blutbahn führt. Da für den Enzymaustritt eine reversible Zellschädigung ausreicht $(5,6,7)$; und weiterhin vor allem in in vitro Versuchen Glukosemangel $(1,3,4,8)$ sowie Stoffwechselgifte $(4,9) \mathrm{zu}$ einer Enzymausschwemmung führen, liegt es nahe, die mangelnde energetische Versorgung der Zelle für den Enzymaustritt verantwortlich zu machen (10). Als Maß für die freie Energie der Zelle kann das Verhältnis der Adeninnucleotide gelten. Wir haben daher zum Teil in Anlehnung an frühere Untersuchungen von BRUNS und Mitarbeitern (11) die Serumenzyme und die freien Adeninnucleotide der Leber bei Ratten nach Vergiftung mit 2,4-Dinitrophenol und Monojodacetat untersucht.

\section{Methodik}

Als Versuchstiere dienten $250-300 \mathrm{~g}$ schwere Wistarratten. Diese erhielten $20 \mathrm{mg} / \mathrm{kg} \mathrm{2,4-Dinitrophenol} \mathrm{i.} \mathrm{p.} \mathrm{in} \mathrm{Form} \mathrm{einer} \mathrm{0,1-proz.}$ Lös. in isotonischer Kochsalzlös. von $37^{\circ}$. Monojodessigsäure gaben wir $60 \mathrm{mg} / \mathrm{kg}$ als 1,2-proz., wäßr. neutralisierte Lösung. In leichtex Äthernarkose wurden zu verschiedenen Zeiten mittels Frierstop Leberproben entnommen. In einem „Mikrodismembrator" der Fa. Braun-Melsungen wurde das Lebergewebe zerkleinert, wobei wir einen aus Teflon selbst angefertigten, etwa $12 \mathrm{~m} /$ fassenden Schüttelbehälter mit einer $15 \mathrm{~mm}$ Stahlkugel verwendeten. Behälter mit Kugel wurden in flüssigem Stickstoff gekühlt, das tiefgekühlte Organmaterial eingefüllt und $1 \mathrm{Min}$. im Gerät zermahlen. Das zerkleinerte Material wurde mit 0,6N Perchlorsäure extrahiert, zentrifugiert und mit $2 \mathrm{~N} \mathrm{KOH}$ neutralisiert. Wir bestimmten ATP mittels Hexokinase und Glukose-6-phosphatDehydrogenase nach LAMPRECHT und Trautschold (13), ADP und AMP mit Pyruvatkinase und Lactatdehydrogenase (Boehringer Test TC-K). Alle Bestimmungen wurden doppelt durchgeführt und die früher angegebene Korrektur vorgenommen (Einzelheiten bei (12)). In den Leberextrakten bestimmten wir ferner den Gehalt an Lactat und Pyruvat enzymatisch mit Lactatdehydrogenase (Boehringer Tests TC-B und TC-C). Bei der Pyruvatbestimmung wie bei der ADP-AMP-Bestimmung verwendeten wir, da der Extrakt bereits neutralisiert war, $0,05 \mathrm{M}$ Triäthanolaminpuffer pH 7,5. Bei der Lactatbestimmung wurde in Abänderung der Testbestimmung ein Leerwert mit Extrakt, jedoch ohne LDH, angesetzt. Bei weiteren Ratten wurde in leichter Athernarkose der Bauchraum eröffnet und durch Punktion der Aorta Blut gewonnen. In dem abzentrifugierten Serum bestimmten wir im optischen Test mit NADH folgende Enzyme: Lactatdehydrogenase (1.1.1.27), Boehringer Test TC-G; Aspartattransaminase (2.6.1.1), Boehringer Test TC-A; Sorbitdehydrogenase (L-Idid DH 1.1.1.14), Boehringer
Test TC-N; Glutamatdehydrogenase (1.4.1.2), Boehringer Test TC-4; in einigen Versuchen ferner Alanintransaminase (2.6.1.2), Boehringer Test TC-H und Malatdehydrogenase (1.1.1.37), Boehringer Test TC-L. Die Glucosephosphatisomerase (5.3.1.9) haben wir nach BRuNs und HinsBerg (14) gemessen durch Bestimmung des gebildeten Fruktose-6-phosphats mit Resorcin. Alle Angaben über Enzymaktivitäten sind einheitlich in Milli-Einheiten $=1 / 1000 \mu \mathrm{Mol}$ Substratumsatz pro Minute pro $\mathrm{m} /$ Serum gemacht.

Um uns über die zur Wirkung kommenden Konzentrationen der Stoffwechselgifte zu informieren, haben wir im Photometer enteiweißtes Serum bei $366 \mathrm{~m} \mu$ gemessen. 2,4DNP hat in alkalischer Lösung ein Absorptionsmaximum bei $360 \mathrm{~m} \mu .1 \mathrm{~m} l$ Serum wurde mit $2 \mathrm{~m} l$ 10-proz. Trichloressigsäure enteiweißt, zu $1 \mathrm{~m} l$ Filtrat wurden $2 \mathrm{ml} 0,5 \mathrm{~N} \mathrm{KOH}$ zugesetzt. Normalserum ohne 2,4-DNP ergibt unter den angegebenen Bedingungen eine Extinktion bei $366 \mathrm{~m} \mu$, die $9,5 \times 10^{-6} \mathrm{Mol} / \mathrm{l} 2,4-\mathrm{DNP}$ entspricht. Da das weniger als $5 \%$ der gemessenen Konzentrationen ist, haben wir diesen Leerwert nicht weiter berücksichtigt.

\section{Ergebnisse}

In den Tabellen 1 und 2 sind die Metabolitgehalte (15) der Leber nach Injektion von 2,4-Dinitrophenol und Monojodacetat zusammengestellt. Die Werte der Adeninnucleotide der Kontrollen stimmen mit den früher von uns veröffentlichten nicht überein (12). Wir fanden damals folgende Werte: ATP: $2,82 \pm 0,24$; ADP: $0,87 \pm$ 0,13; AMP: $0,32 \pm 0,11$ und Summe der Adeninnucleotide: 4,01 $\pm 0,31$. Die jetzigen Normalwerte (Tab. 1 u. 2) liegen für ATP signifikant höher $(p<0,05)$, für ADP und AMP signifikant niedriger $(p<0,01)$; die Summen der Adeninnucleotide unterscheiden sich nur unwesentlich und nicht signifikant. Die Tatsache, daß das Organmaterial mit dem Mikrodismembrator der $\mathrm{Fa}$. Braun viel feiner gemahlen wird, als dies von Hand mit dem Mörser möglich ist und, daß es entsprechend von der Perchlorsäure schneller fixiert wird, dürfte der Grund für das günstigere Verhältnis der Adeninnucleotide sein. $\mathrm{Da}$ für unsere Fragestellung nicht so sehr die Absolutwerte der Adeninnucleotide interessieren, sondern in erster Linie ihr Verhältnis zueinander, haben wir in Abbildung 1 und 2 die Relativprozente für ATP, ADP und AMP gegen die Zeit aufgetragen. Nach Injektion von 2,4-DNP fällt in der Leber das ATP sehr schnell ab, während $\mathrm{ADP}$ und im Verhältnis zum Ausgangswert besonders AMP stark ansteigen. Das Minimum für ATP $=36 \%$, gleichzeitig das Maximum für ADP $=36 \%$ und $\mathrm{AMP}=20 \%$, liegt schon 30 Min. nach der 
Tab. 1. Adeninnucleotide, Lactat und Pyruvat in $\mu \mathrm{Mol} / \mathrm{g}$ Leberfrischgewicht \pm Standardabweichung nach Injektion von 2,4-Dinitrophenol 20 mg/kg Jeder Wert ist Mittelwert aus 6 Einzelwerten. ${ }^{*} p<0,05 ;{ }^{* *} p<0,01 ; * * * p<0,001$

\begin{tabular}{|c|c|c|c|c|c|c|}
\hline & ATP & ADP & AMP & $\begin{array}{l}\text { Summe der } \\
\text { Adeninnucl. }\end{array}$ & Lactat & Pyruvat \\
\hline $\begin{array}{l}\text { Kontrollen } \\
30 \text { Min. } \\
1 \text { Std. } \\
2 \text { Stdn. } \\
4 \text { Stdn. }\end{array}$ & $\begin{array}{ll} & 3,06 \\
\pm & 0,14 \\
1,34 * * * \\
\pm & 0,49 \\
1,76 * * * \\
\pm & 0,42 \\
1,86 * * \\
\pm & 0,86 \\
& 2,39 * \\
\pm & 0,70\end{array}$ & $\begin{aligned} & 0,64 \\
& \pm 0,13 \\
& 1,31 * * * \\
& \pm 0,13 \\
& 1,46 * * * \\
& \pm 0,21 \\
& 1,33^{* *} \\
& \pm 0,23 \\
& 1,03 * * \\
& \pm 0,25\end{aligned}$ & $\begin{array}{ll} & 0,12 \\
\pm & 0,02 \\
1,01 * * * \\
\pm 0,24 \\
0,72 * * \\
\pm 0,27 \\
0,53 * \\
\pm 0,38 \\
0,34 * * * \\
\pm 0,23\end{array}$ & $\begin{array}{r}3,82 \\
\pm 0,30 \\
3,66 \\
\pm 0,21 \\
3,94 \\
\pm 0,43 \\
3,72 \\
\pm 0,33 \\
3,76 \\
\pm 0,57\end{array}$ & $\begin{array}{ll} & 1,07 \\
\pm & 0,37 \\
2,81 * \\
\pm \\
\pm & 1,42 \\
2,04 \\
\pm 1,09 \\
1,51 \\
\pm 0,57 \\
0,76 \\
\pm 0,55\end{array}$ & $\begin{array}{ll} & 0,22 \\
\pm & 0,07 \\
0,17 \\
\pm 0,12 \\
0,09 * * \\
\pm 0,02 \\
0,06 * * * \\
\pm 0,02 \\
0,13 \\
\pm & 0,10\end{array}$ \\
\hline
\end{tabular}

Tab. 2. Adeninnucleotide, Lactat und Pyruvat in $\mu$ Mol/g Leberfrischgewicht \pm Standardabweichung nach Injektion von Monojodacetat 60 mg/kg Jeder Wert ist Mittelwert aus 6 Einzelwerten. ${ }^{*} p<0,05 ; * * p<0,01 ; * * * p<0,001$

\begin{tabular}{|c|c|c|c|c|c|c|}
\hline & ATP & ADP & AMP & $\begin{array}{l}\text { Summe der } \\
\text { Adeninnucl. }\end{array}$ & Lactat & Pyruvat \\
\hline $\begin{array}{l}\text { Kontrollen } \\
2 \text { Stdn. } \\
4 \text { Stdn. } \\
6 \text { Stdn. } \\
8 \text { Stdn. }\end{array}$ & $\begin{array}{r}3,06 \\
+0,14 \\
3,08 \\
+0,45 \\
3,45 \\
+0,45 \\
2,92 \\
+0,67 \\
2,41 \\
+1,14\end{array}$ & $\begin{array}{l}\quad 0,64 \\
\pm 0,13 \\
0,81 \\
\pm 0,25 \\
0,95 * * \\
\pm 0,16 \\
1,25 * * \\
\pm 0,36 \\
1,11 * * \\
\pm 0,33\end{array}$ & $\begin{array}{l}\quad 0,12 \\
\pm 0,02 \\
0,14 \\
\pm 0,01 \\
0,24 * \\
\pm 0,12 \\
0,31 \\
\pm 0,25 \\
0,44 * \\
\pm 0,29\end{array}$ & $\begin{array}{l}3,81 \\
\pm 0,30 \\
4,03 \\
\pm 0,68 \\
4,64 * * \\
\pm 0,43 \\
4,48 * \\
\pm 0,54 \\
3,96 \\
\pm 0,63\end{array}$ & $\begin{array}{ll} & 1,07 \\
\pm & 0,37 \\
0,98 \\
\pm & 0,24 \\
1,56 * \\
\pm 0,17 \\
1,87 * * * \\
\pm 0,69 \\
=2,59 * * \\
\pm 1,74\end{array}$ & $\begin{array}{r}0,22 \\
\pm 0,07 \\
0,22 \\
\pm 0,10 \\
0,24 \\
\pm 0,07 \\
0,20 \\
\pm 0,04 \\
0,21 \\
\pm 0,02\end{array}$ \\
\hline
\end{tabular}
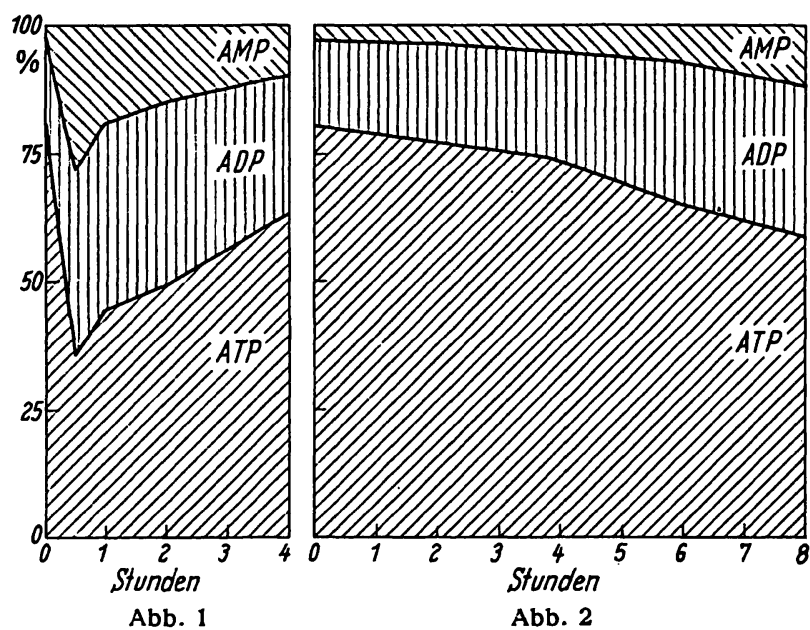

Abb. 1. 2,4-DNP $20 \mathrm{mg} / \mathrm{kg}$ i. p. zur Zeit 0 .

ATP, ADP, AMP in Prozent, Summe der Adeninnucleotide $=100 \%$

Abb. 2. Monojodacetat $60 \mathrm{mg} / \mathrm{kg}$ i. p. zur Zeit 0 .

ATP, ADP, AMP in Prozent, Summe der Adeninnucleotide $=100 \%$

Injektion. Anschließend steigt ATP wieder an, zunächst auf Kosten des AMP und später auch des ADP. Die Summe der Adeninnucleotide verändert sich unter der Wirkung von 2,4-DNP nicht. Die 2,4-DNP-Konzentration im Serum betrug in diesen Versuchen 30 Min. nach Injektion $2,9 \times 10^{-4} \mathrm{Mol} / l$, nach 1 Std. $2,4 \times 10^{-4}$, nach 2 Stdn. $1,7 \times 10^{-4}$ und nach 4 Stdn. $1,3 \times 10^{-4}$ $\mathrm{Mol} / l$. Das sind Konzentrationen, die in vitro vollständig entkoppeln (16).

$\mathrm{Zu}$ der Dosierung von Monojodacetat ist zu bemerken, $\mathrm{da} \beta$ in unseren Versuchen $60 \mathrm{mg} / \mathrm{kg}$ sicher letal wirkten, und zwar innerhalb von $12 \mathrm{Stdn}$. Von den Tieren, die nach 6 und 8 Stdn. getötet werden sollten, starben schon $30 \%$. Trotz dieser hohen Dosierung kommt es, wie aus Abbildung 2 ersichtlich, zunächst in der Leber nicht zu einer wesentlichen Verschiebung des ATP/ADP-Verhältnisses. Dagegen nimmt die Summe der Adeninnucleotide, bezogen auf Leberfrischgewicht, signifikant

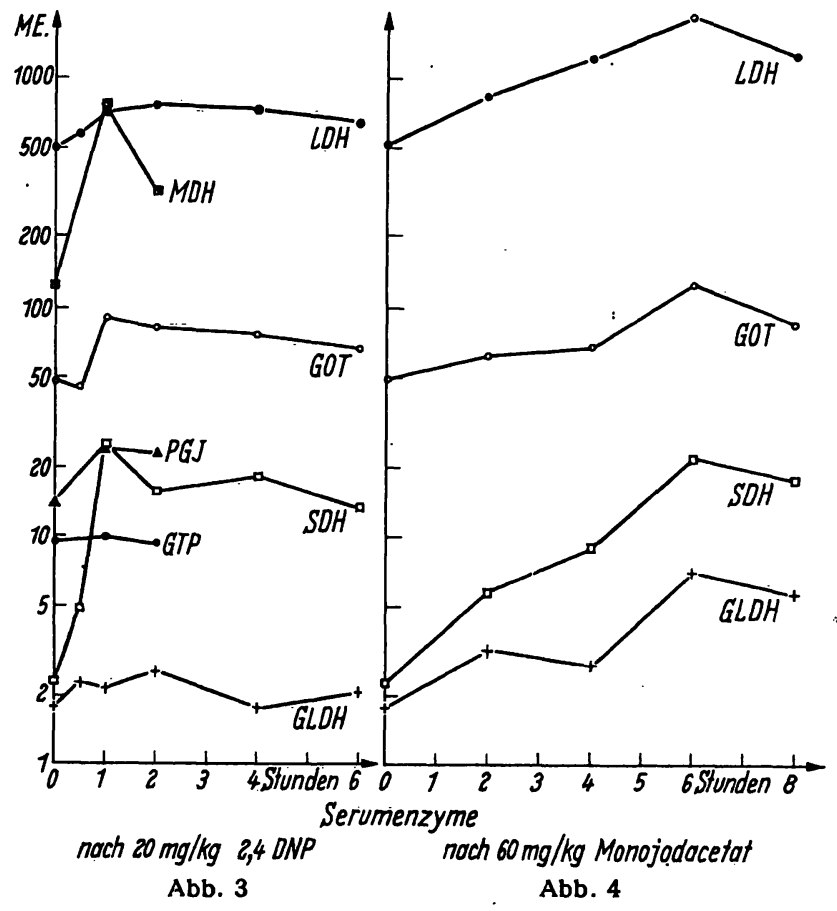

Abb. 3. Serumenzyme nach Injektion von 2,4-DNP $20 \mathrm{mg} / \mathrm{kg}$. Abszisse: Żeit in Stunden nach Injektion,

Ordinate: Enzymaktivitäten in Millieinheiten $/ \mathrm{ml}$ Serum (logarithmisch). LDH: Lactatdehydrogenase; $\mathrm{MDH}$ : Malatdehydrogenase; GOT: Aspartattransaminase; PGI: Glucosephosphatisomerase; SDH: Sorbitdehydrogenase; GPT: Alanintransaminase; GIDH: Glutamatdehydrogenase

Abb. 4. Serumenzyme nach Injektion von Monojodacetat $60 \mathrm{mg} / \mathrm{kg}$; Maßstab und Bezeichnungen wie in Abbildung 3

bis zur 4. Stunde nach Injektion des Stoffwechselgiftes zu. Sie fällt danach wieder $a b$ und erreicht nach 8 Stdn. wieder den Ausgangswert. Dabei kommt es jetzt zu einer Verschiebung des Verhältnisses zu Gunsten von ADP und AMP auf Kosten von ATP, aber nicht entfernt in dem Ausmaß wie unter 2,4-Dinitrophenol.

Sowohl nach 2,4-DNP als auch nach Monojodacetat steigt die Milchsäurekonzentration in der Leber an, als 
Ausdruck eines veränderten Redox-Status, der seinerseits mit der Energetik des Adeninnucleotid-Systems in Zusammenhang steht (17). Nach 2,4-DNP fällt zusätzlich wohl aufgrund einer durch die Entkopplung gesteigerten Oxydation die Pyruvatkonzentration ab. Der Quotient Lactat/Pyruvat, der bei unseren Kontrollen 4,9 beträgt, ist in beiden Fällen deutlich erhöht: Maximum 2 Stdn. nach 2,4-DNP: 25,0 und 8 Stdn. nach Monojodacetat: 12,3 .

Die zeitlichen Veränderungen der gemessenen SerumEnzym-Aktivitäten sind in logarithmischem Maßstab aufgetragen (Abb. 3 u. 4). Gegenüber den Veränderungen der Adeninnucleotide etwa um eine halbe Stunde verzögert, steigen nach Dinitrophenol im Serum die Sorbitdehydrogenase auf das zehnfache, die Malatdehydrogenase auf das siebenfache ihrer Ausgangswerte an. Die übrigen untersuchten Serumenzyme nehmen in ihrer Aktivität nur geringfügig, aber signifikant - die Aspartattransaminase um $87 \%$ und die Glukosephosphatisomerase um $73 \%$ - oder gar nicht $z u$ Glutamatdehydrogenase und Alanintransaminase. Das Maximum der Serumenzymveränderungen in unseren Versuchen mit Monojodacetat liegt bei $6 \mathrm{Stdn}$. Der im Verhältnis zum Ausgangswert größte Anstieg - um etwa das zehnfache - findet sich auch hier für die Sorbitdehydrogenase; die anderen untersuchten Enzyme steigen aber ebenfalls deutlich und signifikant an, einschließlich der Glutamatdehydrogenase. Es fällt auf, daß die Enzymaktivitäten im Serum nach der 6 . Stunde wieder abnehmen, obwohl sich das Verhältnis der Adeninnucleotide in der Leber zwischen der 6. und 8. Stunde weiter verschlechtert.

\section{Diskussion}

Bei der Anlage unserer Versuche gingen wir von der Annahme aus, daß der größte Teil der Stoffwechselgifte nach Resorption vom visceralen Peritoneum in die Leber gelangt. Die relativ starke Vermehrung der weitgehend leberspezifischen Sorbitdehydrogenase haben wir als Bestätigung dieser Annahme aufgefaßit. Entsprechend kommt es nach intraperitonealer Injektion von 2,4Dinitrophenol kurzfristig zum Abfall des ATTP in der Leber. $\mathrm{Da}$ eine Veränderung der Adeninnucleotide von entsprechendem Ausmaß nach einer sicher letalen Dosis Monojodacetat ausbleibt, erscheint nicht so verwunderlich, da andere Organe empfindlicher gegenüber einer Blockade der Glykolyse sein dürften. Die spontan verendeten Tiere starben unter Krampferscheinungen.

Ein erster Vergleich der Abbildungen 1 und 2 mit den Abbildungen 3 und 4 scheint die eingangs zitierte Theorie über den Mechanismus der Enzymausschüttung $(10,11)$ zư bestätigen: in gewissem zeitlichen $\mathrm{Zu}-$ sammenhang $\mathrm{zu}$ den Veränderungen im System der energiereichen Phosphate kommt es zu einem Anstieg verschiedener Serumenzyme. Vergleicht man die Serumenzymveränderungen jedoch mit jenen, die z. B. beim Menschen nach Herzinfarkt oder Hepatitis oder im Tierexperiment nach Tetrachlorkohlenstoff-Vergiftung beobachtet werden, so liegen jene um Größenordnungen böber. Wir können in dieser Hinsicht die schon zitierten Befunde von BRUNS und Mitarbeitern (11) nicht ganz bestätigen, da wir trotz höherer Dosierung der Stoffwechselgifte einen deutlich geringeren Anstieg der Serumenzyme feststellen konnten. In Vorversuchen mit der halben 2,4-DNP-Dosis fanden wir zwar einen geringfügigen ATP-Abfall zugunsten des ADP, aber keinen Anstieg der Serumenzyme, kleinere Dosen Jodacetat waren in jeder Beziehung völlig unwirksam. Wäre die Abnahme der freien Energie der Zelle in jedem Fall die Ursache ihres Enzymverlustes, so sollte man in den Versuchen mit 2,4-DNP - entsprechend der Störung des Energiestoffwechsels - einen wesentlich stärkeren Anstieg der Serumenzyme erwarten. Monojodacetat, das nur eine geringfügige Verschiebung des Verhältnisses ATP/ADP in der Leber hervorruft, bewirkt eine etwa gleich große Enzymausschwemmung wie 2,4-DNP. Ferner sollte man erwarten, daß der Enzymausstrom in die Blutbahn der Energieabnahme zeitlich folgt. Im Falle des Monojodacetats fallen aber die Serumenzymaktivitäten $z$ wischen der 6 . und 8 . Stunde schon wieder $a b$, während das Verhältnis ATP/ADP in der Leber weiter abnimmt. Ist die Abnahme der Serumenzyme zwischen der 6. und 8. Stunde auch nur geringfügig, so spricht doch das gleiche Verhalten aller untersuchten Enzyme gegen Zufälligkeit.

Wie bereits angedeutet, erwarteten wir aufgrund der Versuchsanordnung und aufgrund der relativ starken Vermehrung der Sorbitdehydrogenase ein Enzymverteilungsmuster wie bei einem Leberzellschaden $(17,18)$. Dem entspricht jedoch nicht die gänzlich unveränderte Aktivität der Serumalanintransaminase nach 2,4-DNP. Eine sekundäre Inaktivierung dieses Enzyms konnten wir durch Aktivitätsmessungen nach Zugabe von 2,4DNP in vitro ausschließen. Weiterhin können wir uns das Verhalten der Glutamatdehydrogenase nicht erklären, die nach Monojodacetat deutlich, nach Dinitrophenol aber kaum ansteigt. Weder die unterschiedliche intrazelluläre Lokalisation noch eine von der unterschiedlichen Molekülgröße abhängige Ausflußgeschwindigkeit können diese Verzerrung unseres Erachtens verursachen. Eine ähnliche nicht zu erklärende Verzerrung des Enzymmusters beobachtete Wu (9) in vitro mit Dinitrophenol bei Asciteszellen. Während der Hexokinase- und Aldolase-Efflux in dem Maße zunahm, das dem vermehrten Eiweißverlust nach DNP entsprach, war der Verlust der Zellen an Glycerin-3-phosphatdehydrogenase größer, so daß die spezifische Aktivität im Überstand zunahm. Wu konnte durch Zugabe von Glukose den DNP-Effekt verhindern. Das spricht für eine Stöörung des Energiestoffwechsels als Ursache des vermehrten Enzymaustritts und gegen eine unspezifische Wirkung des DNP. Aufgrund unserer Befunde müssen wir ebenfalls annehmen, daß eine Verminderung des energetischen Potentials die Ursache eines Enzymverlustes der Zelle sein kann, aber nicht in jedem Fall sein muß.

Wir danken Fräulein G. Jungeblodt für fleißige und gewissenhafte Mitarbeit bei der Durchführung der Arbeit. 


\section{Literatur}

1. WARburg, O. und E. Hiepler, Z. Naturforsch. 76,193 (1952). 2. Schade, A. L., Biochim. biophysica Acta (Amsterdam) 12, 163 (1953). - 3. Sibley, J. A., Ann. N. Y. Acad. Sci. 75, 339 (1958). 4. Zierler, K. L., Ann. N. Y. Acad. Sci. 75, 227 (1958). - 5. Henley, K. S., E. Schimidt und F. W. Schmidt, J. Amer. Med. Ass. 173, 977 (1960). - 6. Hess, B. und R. Raftopoulo, Dtsch. Arch. klin. Med. 204, 97 (1957). - 7. Orro, P., E. Schmrdt und F. W. Schmidt, Klin. Wschr. 42, 75 (1964). - 8. WARBURG, O., K. Gawehn und G. LANGe, Z. Naturforsch. 96, 109 (1957). 9. Wu, R., Cancer Res. 19, 1217 (1959). - 10. Hess, B., Enzyme im Blutplasma, Georg Thieme Verlag Stuttgart (1962). - 11. BRUNS, F. H., E. Brosswitz, H. Dennemann, H. D. Horn und E. Nolt-
MANN, Klin. Wschr. 39, 342 (1961). - 12. KRÖNER, H., E. MEINKEN und W. StaIb, diese Z. 5, 81 (1967). - 13. LAMprecht, W. und J. Trautschold, In Methoden der enzymatischen Analyse, Hrsg. H. U. Bergmeyer, Verlag Chemie GmbH, Weinheim/ Bergstr. (1962). - 14. Bruns, F. und K. HINSBERG, Biochem. $Z$. 332, 532 (1954). - 15. Hohorst, H. J., F. H. KreutZ und Th. Bücher, Biochem. Z. 332, 18 (1959). - 16. Hunter, F. E., In Methods in Enzymology, Vol. II, Hrsg. G. P. Colowick u. N. O. Kaplan, Academic Press, New York (1955). - 17. Bücher, TH. und M. Klingenberg, Angew. Chem. 70, 552 (1958). - 18. Delbrück, A., E. Zebe und Th. Bücher, Biochem. Z 331, 273 (1959). - 19. KRöNER, H. und W. StAIB, in Vorbereitung.

Professor Dr. W. Staib, 4 Düsseldorf, Witzelstraße 111

\section{KURZMIT'TEILUNGEN}

\section{Zersetzung des Albuminreagenzes von NESS bei Aufbewahrung in Plastikflaschen}

Von K. KRAMER

Aus dem Klinischen Laboratorium des "Antoni van Leeunenboek-Huis", Amsterdam (Leiter: Dr.J. P. Persijn)

(Eingegangen am 16. Dezember 1966)

Im Jahre 1954 wurde von Rutsters und Mitarbeitern (1) eine Methode zur direkten quantitativen Bestimmung von SerumAlbumin herausgegeben. Sie benutzten hierbei die spezielle Bindung von Albumin an den Farbstoff 2-(4'-Hydroxybenzazo)-Benzoesäure, („HBABA“). 1965 wurde dieses Verfahren von Ness und Mitarbeitern (2) zu einer zuverlässigen Labormethode ausgearbeitet, mit der man gleiche Werte wie mit der Biuretmethode erhält. Angesichts der Zeitersparnis des Ness'schen Verfahrens gegenüber der Biuretmethode wurde dieses in unserem Labor eingeführt. Es stellte sich bald heraus, daß die Haltbarkeit der 6,0 mM HBABALösung von 15 Monaten, welche von Ness angegeben wurde, nicbt zutrifft, wenn die genannte Lösung in Polyäthylenflaschen aufbewahrt wird. Schon nach Verlauf von zwei Monaten verfärbte sie sich und war letzten Endes dunkelbraun bis schwarz. Diese Verfärbung tritt nicht auf, wenn man diese Lösung in Glasflascben aufbewahrt.

Mit Hilfe einer Silicagelsäule konnte eine zwei Monate alte verfärbte Lösung chromatographisch in drei Bänder aufgeteilt werden

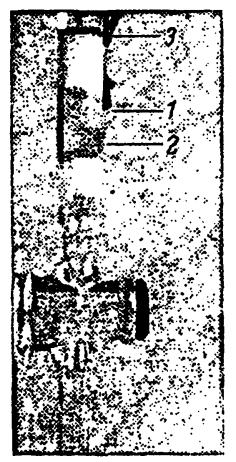

Abb. 1

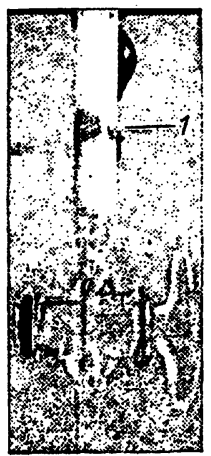

Abb. 2

(Abb. 1). Das erste Band war orange gefärbt, das zweite dunkelbraun und das dritte schwarz-braun. Die im Glasbehälter aufbewahrte Lösung zeigte nur ein Band (Abb. 2). Die Abmessungen der benutzten Silicagelsäure betrugen $40 \times 10 \mathrm{~mm}$. Das Glasrohr wurde mit $2 \mathrm{~g}$ Silicagel in $15 \mathrm{ml}$ Äthylalkohol gefüllt; nachgespült wurde mit reinem Äthylalkohol.

\section{Literatur}

1. Rutstein, D. R., E. F. Ingenito, W. E. Reynolds, und J. M. Burke, J. Clin. Invest. 33, 211 (1954). - 2. Ness, A. T., H. H. Dickerson, und J. V. Pastewka, Clin. Chim. Acta 12, 532 (1965).

K. Kramer, Amsterdam/Holland, Sarphatistraat 106

\section{Diazo-Schnellmethode für die Bestimmung von Gesamtbilirubin mit einem kombinierten Reagenz}

\author{
Von G. HillmanN und G. Beyer
}

Aus dem Chemiscben Institut der Städtischen Krankenanstalten Nürnberg (Vorstand: Prof. Dr. G. Hillmann)

(Eingegangen am 22. Dezember 1966)

Zeitaufwand und Präzision photometrischer Bestimmungsmethoden werden u. a. durch die $\mathrm{Zahl}$ der Pipettierungsvorgänge begrenzt. Die Entwicklung eines stabilen Einwegreagenzes ist bei der zuverlässigen Routine-Diazo-Methode nach JENDRAssIK und Grof (1) nicht möglich. Die Methode von Malloy-Evelyn (2) exfordert eine Verdünnung der Serum/Plasma-Probe wegen des Proteindenaturierungseffektes von Methylalkohol. Dies gilt auch für die von RAND und DI PASQUA vorgeschlagene Modifikation (Ersatz der diazotierten Sulfanilsäure durch diazotiertes 2,4 Dichloranilin) (3).

Wir fanden, daß die Verwendung von Atbylenglykol anstelle von Methylalkohol bei der von RAND und DI PASQUA angewandten Methode zu einem stabilen Einwegreagenz führt, welches nach Zugabe der unverdünnten Serum/Plasma-Probe sowohl im Mikrowie im Ultramikroansatz eine trübungsfreie über mehrere Stunden stabile Extinktion der Azobilirubinverbindungen proportional zum Bilirubingehalt gewährleistet. Äthylenglykol ist nach unseren Versuchen im Gegensatz zu Methylalkohol kein Eiweißfällungsmittel, weist jedoch den gleichen Accelerationseffekt auf die Azobilirubinbildung auf wie Methylalkohol. Das Spektrum der Azobilirubinverbindungen in Athylenglykol unterscheidet sich von der Spektralkurve in Methanol durch eine Verschiebung des Maximums um $5 \mathrm{~m} \mu$, d. h. das Absorptionsmaximum liegt in Methanol bei $540-544 \mathrm{~m} \mu$, in Äthylenglykol bei $545-550 \mathrm{~m} \mu$. Bei der Originalmethode von RAND und DI PASQUA ist die Farbreaktion über 30-40 Min. stabil, bei Verwendung von Äthylenglykol über 5 Stdn. Die photometrische Messung erfolgt nach mindestens 2-minütiger Farbentwicklung bei $550 \mathrm{~m} \mu$.

Für den Routine-Mikroansatz werden $0,2 \mathrm{ml}$ hämolysefreies Serum oder Plasma verwendet, bei stark ikterișchen Seren $0,1 \mathrm{ml}$. Wegen der hohen Viscosität des Diazo-Reagenzes kann bei Ultramikroansätzen eine automatische Absaugevorrichtung nicht verwendet werden.

Arbeitsvorschrift

\section{Reagenzien}

$0,01 \mathrm{M} 2,4-$ Dichloranilin in $0,25 \mathrm{~N} \mathrm{HCl}$. $0,1 \mathrm{M} \mathrm{NaNO}_{2}$ in Ẅasser.

Äthylenglykol (Äthandiol-1,2), Fa. Merck.

Diazo-Reagenz A: $50 \mathrm{ml}$ Dichloranilinlösung werden mit $1 \mathrm{~m} l$ Nitritlösung gemischt und $15 \mathrm{Min}$. im Kühlschrank bei $6^{\circ}$ aufbewahrt, anschließend Zugabe von $250 \mathrm{ml}$ Äthylenglykol. Haltbarkeit: etwa $12 \mathrm{Stdn}$. bei Zimmertemperatur, etwa $48 \mathrm{Stdn}$. bei $6^{\circ}$. 